\title{
Mixed-ethnicity face shape and attractiveness in humans
}

\author{
Anthony C Little ${ }^{1}$, Kimberley J Hockings ${ }^{1,2}$, Coren L Apicella ${ }^{3}$, Claudia Sousa ${ }^{2,4}$ \\ ${ }^{1}$ School of Natural Sciences, University of Stirling, Stirling FK9 4PA, UK; \\ e-mail: anthony.little@stir.ac.uk; ${ }^{2}$ Centre for the Research in Anthropology (CRIA), Faculdade de \\ Ciências Sociais e Humanas [ ${ }^{4}$ Departamento de Antropologia], Universidade Nova de Lisboa, \\ Av. Berna, 26-C, 1069-061 Lisbon, Portugal; ${ }^{3}$ Department of Psychology, University of \\ Pennsylvania, Philadelphia, PA 19104, USA \\ Received 2 May 2012, in revised form 6 August 2012
}

\begin{abstract}
Many studies show agreement within and between populations and cultures for general judgments of facial attractiveness. Studies that have examined the attractiveness of specific traits have also highlighted cross-cultural differences for factors such as symmetry, averageness, and masculinity. One trait that should be preferred across cultures is heterozygosity. Indeed, several studies suggest that mixed ethnicity, in terms of appearing to possess a mixture of traits from different human population groups, may be found attractive, which could reflect preferences for heterozygosity. We examined preferences for manipulated face shape associated with different populations in both Europeans (Britain) and Africans (Guinea-Bissau). We found that mixed-ethnicity face shapes were more attractive than enhanced single-ethnicity face shape across both populations. These results are consistent with evolutionary theories suggesting individuals should prefer heterozygosity in partners because facial cues to mixed-ethnicity are likely to indicate diverse genes compared to cues that indicate a face belongs to a single particular culture or population.
\end{abstract}

Keywords: facial attractiveness, mixed, culture, population, agreement, variation

\section{Introduction}

An evolutionary view of human facial attractiveness posits that certain traits are indicators of mate value, the degree to which individuals could enhance the fitness of their partners. These traits may indicate good health, fecundity, physical, or behavioural dominance, and even pro-social traits or investment (Little et al 2011). It might then be expected that if some cues are reliably associated with mate value that there would be universal agreement on which faces are attractive and unattractive and, indeed, across many studies considerable agreement is found within a culture as well as across different populations and cultures (see Langlois et al 2000, for a meta-analytic review). For example, Asian and Hispanic students in the USA agree with White Americans on the rated attractiveness of Asian, Hispanic, Black, and White women, and these ratings also positively correlate with ratings made by Taiwanese participants of the same faces (eg Cunningham et al 1995). Such studies have generally only examined agreement on global attractiveness (ie is one face more attractive than another face) and such studies have usually examined urban university-based populations. Of course, a truly adaptive view of mate choice posits different preferences according to condition or context, which leads to individual differences and there is ample evidence for such variation within particular populations and cultures. Recent studies examining preferences for specific traits, such as masculinity and symmetry, have demonstrated some similarities alongside population and cultural differences in preferences (Apicella et al 2007; DeBruine et al 2010; Little et al 2007; Penton-Voak et al 2004).

Preferences for genetic diversity in a partner are an interesting case in mate choice. Because each individual has a unique set of genes, individuals will have different genes in common with others and so preferences may depend on individual variation. This then 
can lead to individual differences in preferences, but also to population level preferences for cues to genetic diversity. There are two main factors related to preferences for cues to genetic diversity: genetic similarity between individuals and heterozygosity/homozygosity. Selecting mates who are more genetically dissimilar to oneself should lead to offspring which are genetically diverse and be more likely to be heterozygous as genes are randomly mixed. Selecting heterozygous partners, individuals who have two different alleles of a gene, also increase the chances that offspring will be heterozygous rather than homozygous. Preferences based on genetic similarity and heterozygosity are related to issues of inbreeding and outbreeding with the prediction that genetic diversity should be preferred in mates because of potential health benefits to resulting offspring (Brown 1997).

In many non-human animals heterozygotes have advantages in terms of producing more offspring and having higher fertility (Charpentier et al 2005; Gomendio et al 2000), and homozygosity is related to inbreeding and its negative consequences (Bittles and Neel 1994). For example, in mandrills, male reproductive success increases with heterozygosity and diversity of the major histocompatibility complex (Setchell et al 2010). When examining genetic similarity between mothers and sires in mandrills, a given male is more likely to have sired the offspring if their similarity with the mother was lower (Setchell et al 2010). Similar behavioural adaptations suggesting preferences for heterozygosity and outbreeding are seen in other species. In bird species, for example, it has been found that females who are more genetically similar to their partners tend to have more extra-pair offspring (Tarvin et al 2005), and that offspring sired by extra-pair mates are more heterozygous because of lower genetic similarity between females and their extra-pair mates (Suter et al 2007). Heterozygous extrapair offspring were also found to be heavier and larger and have higher fledgling survival compared with within-pair offspring (Suter et al 2007). Outbreeding can also have negative consequences, however, such as causing the disruption of locally selected gene complexes (Marshall and Spalton 2000). Indeed, there may be an optimal level of outbreeding/inbreeding which balances the advantages/disadvantages of each (Bateson 1980).

In humans, negative outcomes of having offspring with closely related individuals include higher neonatal and infant mortality and higher incidences of single-gene disorders (Bittles and Neel 1994). Humans do show preferences for the faces of men who are heterozygous at the major histocompatibilty complex (MHC), genes involved in coding for immune function, rating them as more attractive than homozygous men (Roberts et al 2005). Within relationships, women who share a greater proportion of MHC alleles report more extra-pair sexual partners and are more attracted to men other than their primary partners at peak fertility than women who share fewer alleles, suggesting a preference in favour of outbreeding (Garver-Apgar et al 2006). Finally, it appears that humans may have evolved other adaptations designed to prevent inbreeding, such as the Westermarck effect, by which individuals avoid mating with individuals they have shared their childhood with (Westermarck 1894; Wolf 1993).

One arena in which inbreeding/outbreeding might be observed is in preferences for individuals of the same versus different populations. Individuals from other populations are more likely to possess different genes than those from the same population, although genetic differences may be relatively small (Jorde and Wooding 2004). There is limited work in this area; however, two studies have addressed preferences for individuals from the same or different populations, with a focus on whether individuals of mixed race are particularly attractive (Lewis 2010; Rhodes et al 2005). These studies use the term "race" to refer to the physical differences in facial appearance visible between human populations and cultures, and to classify individuals as belonging to different populations and cultures. We adopt the term "ethnicity" to denote both visible phenotypic appearance and distinguish our two populations here because use of the term race is controversial (Jorde and Wooding 2004). We also note that "ethnicity" is often used to refer to shared cultural values rather than 
phenotypic appearance or to distinguish between populations and that our use of the term is broad, encompassing countries in which there are multiple ethnic groups. Both Rhodes et al (2005) and Lewis (2010) found that images of individuals identified as mixed ethnicity were rated as more attractive than those of individuals who were predominantly identified as belonging to one ethnic grouping. Rhodes et al (2005) examined individuals with Asian, European, and mixed Asian-European backgrounds, though the sample size was small, while Lewis (2010) examined male and female individuals with African, Caucasian, and mixed African-Caucasian backgrounds, and with a large sample size. Mixed-ethnicity faces then appear attractive across studies, across sex of face, and across different mixtures of populations.

Both of the above studies postulate that preferences for mixed-ethnicity faces might represent attraction to heterozygosity, and Lewis (2010) particularly draws attention to heterosis, or hybrid vigour. Heterosis refers to the phenomenon whereby cross-breeding leads to organisms that appear to be particularly hardy, healthy, or successful (Duvick 2001; Lippman and Zamir 2007). For example, in non-human animals, cross-bred dairy cows gain weight faster and produce more milk (Cundiff et al 1974) and cross-bred dogs are more successful in guide dog training (Ennik et al 2006). In humans, heterosis has been suggested to be linked to factors such as early growth (Penrose 1955; Wolanski et al 1970) and some authors have suggested that increase in IQ may be linked to heterosis (Mingroni 2007). Such arguments might suggest that mixed-ethnicity faces may be attractive because such individuals have different appearing faces (eg healthier, more symmetric, etc) because individuals with a diverse set of genes are able to develop or maintain attractive traits. In this line of thinking, mixed ethnicity itself may not be what is preferred, but rather the preference is for other traits that are associated with heterozygosity resulting from mixed ethnicity. Previous studies showing preferences for the faces of mixed-ethnicity individuals may reflect preferences for the outcome of heterozygosity (through heterosis), but might also reflect preferences for phenotypic/morphological cues to heterozygosity itself. In other words, there remains a question of whether preferences for mixed-ethnicity faces reflect pressures to simply select attractive mates, which may be influenced by heterozygosity, or whether cues to heterozygosity are themselves attractive.

Rhodes et al (2005) also demonstrated that artificially manipulated faces that possessed mixed-ethnicity shape and colour were more attractive than faces belonging to a single ethnicity by presenting computer images varying along a continuum between European and Asian. This suggests mixed-ethnicity faces are preferred when controlling for any direct increase in attractiveness due to genetic diversity. Preferences for averageness have also been thought to reflect preferences for genetic diversity (Thornhill and Gangestad 1993) and individuals who are heterozygous at the MHC also have more average-appearing faces (Lie et al 2008). Mixed-ethnicity preferences may then also reflect preferences for averageappearing faces (Langlois and Roggman 1990), though this would likely be dependent on exposure to faces of different ethnicities (Apicella et al 2007).

In the current study, we further examined the idea of visual preferences for faces across different populations and cultures. Previous work focusing on unmanipulated single-ethnicity versus mixed-ethnicity faces cannot distinguish between preferences for the outcome of heterozygosity (eg heterozygosity leading to increased observable phenotypic health) or direct preferences for heterozygosity/averageness (eg perceivers use mixed-ethnicity appearance as a cue to heterozygosity). Work using facial manipulations can directly examine effects that are to do with perception as they are control for the effects of heterozygosity itself on facial appearance. One previous study using manipulations has examined individuals of Asian versus European decent demonstrating mixed-ethnicity preferences (Rhodes et al 2005). 
It is important to examine other cross-cultural comparisons to determine whether such effects are specific to certain faces and are more widely applicable. Here we examined individuals of African versus European decent as a visually distinct comparison from Asian versus European decent. Here we also focus on shape-only rather than shape and colour differences between faces. While colour may be a useful cue to mixed-ethnicity status, it is also variable according to tanning practices and sunlight exposure. This is an issue for previous research, as mixing Asian and European faces will lighten Asian faces and darken European faces. If populations and cultures differ in how much they value skin lightness, then this could generate preferences for mixed-ethnicity faces if, for example, European observers value darker skin while Asian observers value lighter skin. Indeed, skin bleaching is common in some African countries and motivations include appearing more European looking (Lewis et al 2011).

We presented European participants from Britain and African participants from GuineaBissau with manipulated face pairs. Participants were presented with pairs of same-ethnicity images that were transformed on the basis of the shape difference between European and African faces. Images represented pairs in which one image possessed enhanced typical European facial shape and one image possessed enhanced typical African facial shape. For Europeans, for example, looking at European faces, selecting the plus-European face as more attractive would represent a preference for European faces with enhanced typical European features (an own-ethnicity typicality preference), whereas selecting the plusAfrican face as more attractive would represent a preference for European faces with less typical European features (a mixed-ethnicity preference).

\section{Methods}

\subsection{Participants}

174 European participants reporting to be white and from the UK ( 88 males, 86 females, aged $16-60$ years, mean age $=28.9$ years, $\mathrm{SD}=10.8$ years) and 111 African participants from the Guinea-Bissau (57 males, 54 females, aged 16-60 years, mean age $=31.3$ years, $\mathrm{SD}=10.5$ years) took part in the study. European participants were selected for reporting to be heterosexual. Sexual orientation questions were omitted for the Guinea-Bissau participants because this was deemed to be sensitive and not culturally appropriate. All participants were volunteers. The study was conducted in line with the Declaration of Helsinki and the British Psychological Association's ethical guidelines.

The Republic of Guinea-Bissau lies on Africa's north-western coast and covers an area of $13948 \mathrm{~km}^{2}$. Data were collected in Cantanhez National Park (Hockings and Sousa 2011), located in the south-western part of Guinea-Bissau, in the Tombali Administrative Region (see map, figure $\mathrm{A} 1$, in the appendix): northeast limit: $11^{\circ} 22^{\prime} 58^{\prime \prime} \mathrm{N}, 14^{\circ} 46^{\prime} 12^{\prime \prime} \mathrm{W}$; southwest limit: $\left.11^{\circ} 2^{\prime} 18^{\prime \prime} \mathrm{N}, 15^{\circ} 15^{\prime} 58^{\prime \prime} \mathrm{W}\right)$. Within the borders of the $1057 \mathrm{~km}^{2}$ Cantanhez National Park there are 110 villages (locally known as 'tabancas'), and a recent population census estimates that 22505 people live there with a population density of approximately 20 people per $\mathrm{km}^{2}$. There are numerous ethnic groups in Guinea-Bissau including Balanta (30\%), Fula (20\%), Manjaco (14\%), Mandinga (13\%), Papel (7\%) (Central Intelligence Agency 2012). A map of the region can be seen in the Electronic Appendix. Guinea-Bissau has a high population growth rate $(1.97 \%)$, around $70 \%$ of the population lives in rural areas with the majority of livelihoods being agricultural, and, in terms of literacy, $42.4 \%$ of the population at age 15 plus can read and write (Central Intelligence Agency 2012).

\subsection{Stimuli}

Original photographs were from larger sets of images taken under standardised lighting in the UK (60 male and 60 female) and unstandardised lighting in Guinea-Bissau (64 male and 55 female). All participants were instructed to look directly at the camera with a neutral 
expression and were all of young adult appearance. Six composite male and six composite female face images were created for each population made from 5 original photographs each from the sets described above (using 30 male and 30 female images from each set). All images were manipulated to match the position of the left and right eyes by standardising inter-pupillary distance and transformed so that the position of the features on either side of the face was symmetrical. Composite images were made to generate the plus-European and plus-African images, and original images were transformed on the basis of the difference between two composite images. We applied a shape-only $\pm 50 \%$ transform using the average European composite and average African composite face of the appropriate sex. This created two images for each starting face, a plus-European face and a plus-African face. Images maintained original textural cues and were symmetric in shape alone (ie faces retained any asymmetries in face colour). See Perrett et al (1998), Rowland and Perrett (1995), and Tiddeman et al (2001) for technical details. An example of manipulated faces can be seen in figure 1.

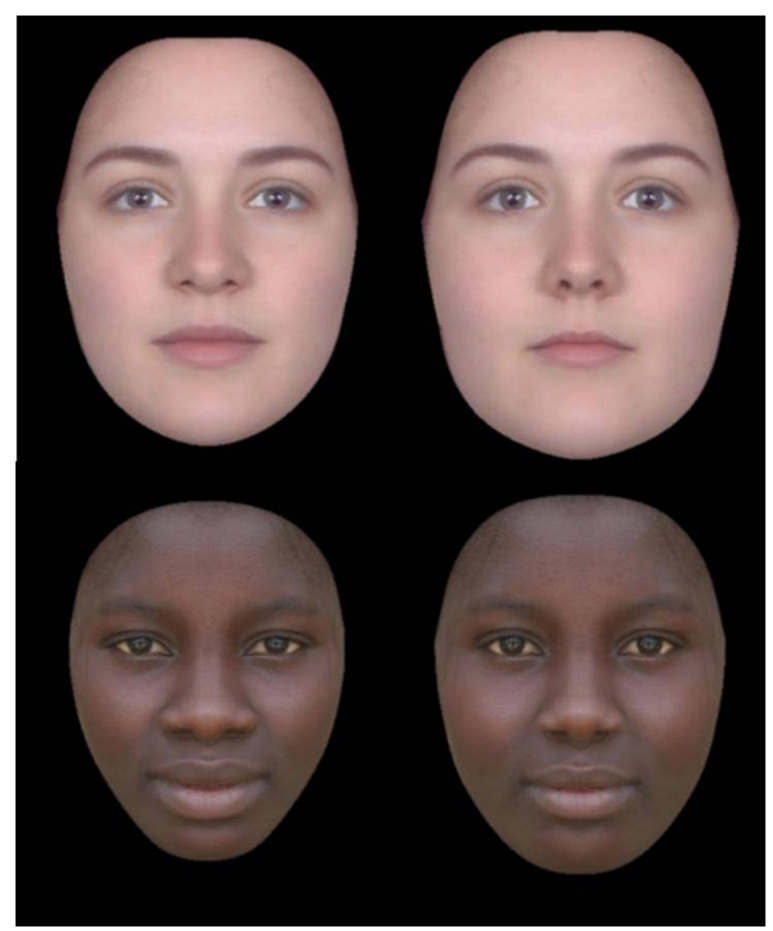

Figure 1. [In colour online, see http://dx.doi.org/10.1068/p7278] Examples of plus-African (left) and plus-European (right) European female and African female faces.

\subsection{Procedure}

All participants were presented with face pairs of the opposite sex. European and GuineaBissau individuals participated in different ways though the instructions and images were equivalent. The Europeans had the faces presented electronically on a computer screen and the test was self-administered. The Guinea-Bissau participants had the test administered to them on photographic quality printed cards $(4.6 \mathrm{~cm} \times 5.8 \mathrm{~cm})$ by the second author and a local research assistant/translator (RA). The RA introduced the experimenter to the participants and gave a brief description of what would be asked of them during the experiment. Tests were administered in Creole, a mixture of Portuguese and regional dialects, spoken throughout the country, and, if necessary, the RA translated instructions into the local language. Each ethnic group has its own language, however, and the country's official language is Portuguese (Central Intelligence Agency 2012). 
Both Europeans and Guinea-Bissau participants had unlimited time to complete their judgments. The face pairs were presented in random order with participants being asked to choose from the pair: "which face is most attractive?". For each trial, presentation of plusEuropean/plus-African on left or right was randomised. Age, sex, and sexual orientation were recorded in a short questionnaire for the European participants and the experimenter noted sex and age for the Guinea-Bissau participants. Randomisation for the Europeans was done using computer-generated random numbers, whereas for the Guinea-Bissau participants this was done via the experimenter shuffling the image pairs and dealing left and right images at random. For the Guinea-Bissau participants, only the experimenter and the participant were present during testing.

\section{Results}

We computed preference for plus-European faces as the number of plus-European faces chosen converted to a percentage score $(100 \%=$ all plus-European faces chosen). This was computed separately for European and African faces.

A repeated-measures ANOVA was conducted with 'face ethnicity' (European/African) as a within participant factor, and 'rater ethnicity' (European/African) and 'rater sex' (male/ female) as between-participant factors. This revealed a significant main effect of face ethnicity $\left(F_{1,281}=300.55, p<0.001, \mu^{2}=0.517\right)$, an overall significant effect of rater ethnicity $\left(F_{1,281}=39.52, p<0.001, \mu^{2}=0.123\right)$, a significant interaction between face ethnicity and rater ethnicity $\left(F_{1,281}=30.43, p<0.001, \mu^{2}=0.098\right)$, and a three-way interaction amongst rater sex, face ethnicity, and rater ethnicity $\left(F_{1,281}=4.25, p=0.040, \mu^{2}=0.015\right)$. No other effects or interactions were significant (all $F_{1,281}<0.68, p>0.412, \mu^{2}<0.002$ ). Adding age as a covariate did not change this pattern of results, although the three-way interaction above became non-significant $\left(F_{1,280}=3.40, p=0.066, \mu^{2}=0.012\right)$. Additionally, there was a significant interaction between age and face ethnicity $\left(F_{1,280}=4.49, p=0.035, \mu^{2}=0.016\right)$. Age was nonsignificantly positively correlated with preferences for plus-European transforms in African faces $(r=0.083, p=0.164)$ and negatively correlated with preferences for plus-European transforms in European faces $(r=-0.041, p=0.487)$. Together this suggests a weak effect, whereby older participants were more likely to prefer faces transformed toward the other ethnicity.

From figure 2 it can be seen that European judges had stronger preferences for plus-African transforms than African judges, reflecting the main effect of rater ethnicity. We conducted separate ANOVAs for each rater ethnicity to examine the interactions. For African raters, this again revealed a significant main effect of face ethnicity $\left(F_{1,109}=54.56\right.$, $p<0.001, \mu^{2}=0.334$ ) and a close to significant interaction between face ethnicity and rater sex $\left(F_{1,109}=3.07, p=0.083, \mu^{2}=0.027\right)$. No significant effect of rater sex was seen $\left(F_{1,109}=0.01\right.$, $\left.p=0.905, \mu^{2}<0.001\right)$. For European raters, this again revealed a significant main effect of
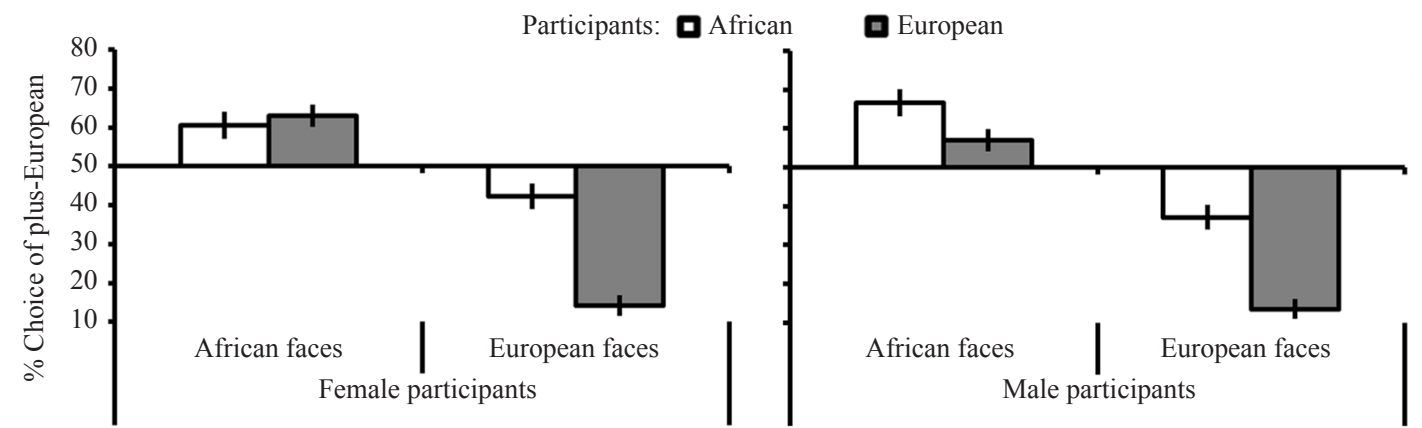

Figure 2. Preferences for plus-European transforms ( $\pm 1 \mathrm{SE}$ of mean) in opposite-sex faces split by male and female participants and European and African judges. 
face ethnicity $\left(F_{1,172}=345.96, p<0.001, \mu^{2}=0.668\right)$. There was no significant interaction between face ethnicity and rater $\operatorname{sex}\left(F_{1,172}=1.15, p=0.285, \mu^{2}=0.007\right)$ and no significant effect of rater $\operatorname{sex}\left(F_{1,172}=1.69, p=0.195, \mu^{2}=0.010\right)$. The three-way interaction from the first analysis reflects that men in the African sample show a stronger effect in preferring mixed-ethnicity faces than do African women and that this is not true for the European participants.

We additionally conducted separate ANOVAs for ethnicity and sex of rater. For female African $\left(F_{1,53}=14.57, p<0.001, \mu^{2}=0.216\right)$, male African $\left(F_{1,56}=45.55, p<0.001\right.$, $\left.\mu^{2}=0.449\right)$, female European $\left(F_{1,85}=169.35, p<0.001, \mu^{2}=0.666\right)$, and male European $\left(F_{1,87}=177.93, p<0.001, \mu^{2}=0.672\right)$ there was a significant effect of face ethnicity.

Separate one-sample $t$-tests comparing preferences for plus-European against chance $(50 \%)$ for each face ethnicity revealed significant preferences for plus-African in the European faces for both European men $\left(t_{87}=16.18, p<0.001\right.$, Cohen's $\left.d=3.47\right)$ and women $\left(t_{85}=16.46, p<0.001\right.$, Cohen's $\left.d=3.57\right)$ and African men $\left(t_{56}=3.04, p=0.004\right.$, Cohen's $d=0.81)$ and women $\left(t_{53}=2.14, p=0.037\right.$, Cohen's $\left.d=0.59\right)$. These tests also revealed significant preferences for plus-European in the African faces for both the European men $\left(t_{87}=2.30, p=0.024\right.$, Cohen's $\left.d=0.49\right)$ and women $\left(t_{85}=5.17, p<0.001\right.$, Cohen's $\left.d=1.12\right)$ and African men $\left(t_{56}=4.71, p<0.001\right.$, Cohen's $\left.d=1.26\right)$ and women $\left(t_{53}=2.84, p=0.006\right.$, Cohen's $d=0.78)$.

To examine generalisation across faces, we split the sample by sex of judge and calculated the mean score given to each face seen (6 African and 6 European). Scores for the European faces were reverse-scored such that higher scores indicated preferences for faces of the other ethnicity (ie high scores indicated preferences for African-like European faces and Europeanlike African faces). One-sample $t$-tests revealed significant preferences for plus other-ethnicity transforms over same-ethnicity transforms for both women judging male faces $\left(t_{11}=4.54\right.$, $p=0.001$, Cohen's $d=2.74)$ and men judging female faces $\left(t_{11}=5.09, p<0.001\right.$, Cohen's $d=3.07)$.

\section{Discussion}

The current study demonstrated similarities in face preferences between African and UK judges for opposite-sex faces. Preferences for plus-European transforms varied according to the starting ethnicity of the faces. Both men and women in both populations preferred plus-European transforms when the images were African and plus-African transforms when the images were European. This suggests that participants were more averse to faces with exaggerated own-face ethnicity traits, and instead preferred what could be described as mixed-ethnicity face shapes. In other words, participants preferred face shapes that were between the two types of face ethnicity presented here. The methods of administration differed between the two groups, with one group taking the test with the experimenter present and the other group taking the test on computer screen. There is, however, no strong reason to believe that participant's choices or motivations would differ with the manner in which the test was carried out.

Our findings are consistent with previous research demonstrating faces of individuals with mixed ethnic backgrounds are rated as more attractive than individuals from single ethnicities (Lewis 2010; Rhodes et al 2005). However, our studies focus on manipulations of ethnicity rather than real mixed ethnicity, thereby controlling for any potential increase in attractiveness due to genetic diversity put forward to explain preferences for mixed-ethnicity faces (Lewis 2010). In our images only shape was manipulated in the faces and other factors were held constant. Given cross-cultural differences in skin colour preferences (Lewis et al 2011), changing both shape and colour is a potential issue for the only other previous study showing preferences for mixed ethnicity in manipulated faces (Rhodes et al 2005). 
We then replicate and extend the findings of Rhodes et al (2005) to another cross cultural comparison controlling for colour. Preferences for mixed ethnicity in individuals who belong to a particular population might reflect preferences for heterozygosity or genetic diversity leading to choice of partners who are healthy and disease resistant and/or avoiding mating with individuals with deleterious mutations. Such a preference may also lead to gaining the advantages of outbreeding (Charpentier et al 2005; Gomendio et al 2000) and avoiding the negative consequences of inbreeding (Bittles and Neel 1994) for offspring.

Our transforms reflect plus-European/African versus mixed ethnicity and so both types of image were equidistant from average faces of the population. In terms of ultimate causation, preferences for averageness (Langlois and Roggman 1990) have also been thought to reflect selection pressures to choose mates who are genetically diverse (Thornhill and Gangestad 1993). At the proximate level, because individuals who are heterozygous at the MHC also have more average-appearing faces (Lie et al 2008), selection of average faced partners will lead to selection of heterozygous partners. While our results might reflect choosing faces closest to an overall population average of both face types as most attractive, such an interpretation requires that all faces encountered are used in building a single prototypic representation. Faces of different ethnicities appear, however, to be processed in a categorical fashion (Jaquet et al 2008; Little et al 2008). For example, it is possible to manipulate the perceived normality of African and European faces to European observers independently, suggesting some level of discrete mental representation (Little et al 2008). If European and African faces are processed somewhat independently, then comparison to an average is unlikely to account for preferences for plus-European/African transforms in European faces here. Even if the effects here do represent attraction to averageness, rather than mixed ethnicity, the logic of why such preferences could be adaptive remains true, such as preferences for heterozygosity resulting in increased health of partners and/or promoting outbreeding.

In terms of outbreeding, the diverse groups of Europeans and Africans are on a continuum of genetic similarity. There are, of course, many more similarities than differences, but ethnicity here presents a useful vector to define differences, such as they are, present in facial shape as a cue to similarity. It is unlikely that our results are specific to comparing between ethnicities; it appears more likely that the effects seen here may apply generally to gradations of relatedness. In this way mixed-ethnicity preferences may arise from mechanisms linked to choosing amongst differently related individuals within a particular population and not reflect selection based specifically on differences in ethnicity.

There were some specific effects of ethnicity of observer interacting with sex of observer as well as an overall effect of ethnicity. A three-way interaction indicated that men in the African sample demonstrated a stronger effect in preferring mixed-ethnicity faces than do African women while effects were equally strong for men and women in the European participants. Such a specific effect is difficult to interpret. In our study, sex of judge and sex of stimuli are confounded, and so the effect could reflect differences in either male and female stimuli or male and female judges. At the stimuli level, the transforms may not be of equivalent salience for male and female faces. The result could then reflect that the transform was more salient in female faces particularly to African men. At the level of judge, the finding could alternatively reflect different pressures on partner choice in the African sample, such that selection of partners with diverse genes is of greater importance to men than women. Additionally, because in this African population marriage choices of women tend to be made by older men (Frazao-Moreira 2009), the African women may have been less engaged in preference tasks involving men. Interestingly, at least for the African sample, stronger preferences for mixed-ethnicity transforms in female than male stimuli is consistent 
with previous findings, demonstrating that in real images of mixed-ethnicity versus singleethnicity individuals that a mixed-ethnicity advantage in terms of attractiveness is stronger for female faces than male faces (Lewis 2010).

There was also a large effect of ethnicity on preference, with European participants most strongly preferring faces of mixed ethnicity, although this appeared mainly for European faces. It is possible that greater visual exposure to a mixture of ethnicities in the European sample could affect their preferences. It is also possible that European participants valued potential genetic diversity associated with mixed ethnic shape more so than the African participants.

In summary, the current study demonstrates preferences for faces falling between human populations differing in phenotypic appearance in both European and African participants. Both samples preferred face images transformed to have traits more typical of the other face type (more European-like African faces and more African-like European faces) than faces that exaggerated same face type traits (more European-like European faces and more African-like African faces). Evolutionary relevant preferences for heterozygosity, or average combinations of genes, may play a role in generating such similarities in preferences between different populations and cultures.

Acknowledgments. This work was supported by Royal Society University Research Fellowship to Anthony Little, a post-doctoral research grant (SFRH/BPD/38595/2007) to Kim Hockings, and research grant (PPCDT/ANT/57434/2004) to Claudia Sousa from Fundação para a Ciência e a Tecnologia, Portugal. We would like to thank the local assistants in Guinea-Bissau for their invaluable help and villagers for continuing support. We are grateful to T Rodriguez and A Barata for fieldwork support in Guinea-Bissau.

\section{References}

Apicella C L, Little A C, Marlowe F W, 2007 "Facial averageness and attractiveness in an isolated population of hunter-gatherers" Perception 36 1813-1820

Bateson P, 1980 "Optimal outbreeding and the development of sexual preferences in Japanese Quail" Zeitschrift für Tierpsychologie 53 231-244

Bittles A H, Neel J V, 1994 "The costs of human inbreeding and their implications for variations at the DNA level" Nature Genetics 8 117-121

Brown J L, 1997 "A theory of mate choice based on heterozygosity" Behavioral Ecology 8 60-65

Central Intelligence Agency, 2012, https://www.cia.gov/library/publications/the-world-factbook/geos/ pu.html

Charpentier M, Setchell J M, Prugnolle F, Knapp L A, Wickings E J, Peignot P, Hossaert-McKey M, 2005 "Genetic diversity and reproductive success in mandrills (Mandrillus sphinx)" Proceedings of the National Academy of Sciences of the USA 102 16723-16728

Cundiff L V, Gregory K E, Koch R M, 1974 "Effects of heterosis on reproduction in Hereford, Angus and Shorthorn cattle" Journal of Animal Science 38 711-727

Cunningham M R, Roberts A R, Barbee A P, Druen P B, 1995 " "Their ideas of beauty are, on the whole, the same as ours': Consistency and variability in the cross-cultural perception of female attractiveness" Journal of Personality and Social Psychology 68 261-279

DeBruine L M, Jones B C, Crawford J R, Welling L L M, Little A C, 2010 "The health of a nation predicts their mate preferences: cross-cultural variation in women's preferences for masculinized male faces" Proceedings of the Royal Society of London B 277 2405-2410

Duvick D N, 2001 "Biotechnology in the 1930s: the development of hybrid maize" Nature Reviews Genetics 2 69-74

Ennik I, Liinamo A E, Leighton E, Arendonk J van, 2006 "Suitability for field service in 4 breeds of guide dogs" Journal of Veterinary Behavior - Clinical Applications and Research $167-74$

Frazao-Moreira A, 2009 Plantas e "Pecadores". Percepções da Natureza em África (Lisbon: Livros Horizonte) 
Garver-Apgar C E, Gangestad S W, Thornhill R, Miller R D, Olp J J, 2006 "Major histocompatibility complex alleles, sexual responsivity, and unfaithfulness in romantic couples" Psychological Science $17830-835$

Gomendio M, Cassinello J, Roldan E R S, 2000 "A comparative study of ejaculate traits in three endangered ungulates with different levels of inbreeding: fluctuating asymmetry as an indicator of reproductive and genetic stress" Proceedings of the Royal Society of London B 267 875-882

Hockings K J, Sousa C, 2011 "Human-chimpanzee sympatry and interactions in Cantanhez National Park, Guinea-Bissau: current research and future directions" Primate Conservation 26 1-9

Jaquet E, Rhodes G, Hayward W G, 2008 "Race-contingent aftereffects suggest distinct perceptual norms for different race faces" Visual Cognition 16 734-753

Jorde L B, Wooding S P, 2004 "Genetic variation, classification and 'race' " Nature Genetics 36 S28-S33

Langlois J H, Kalakanis L, Rubenstein A J, Larson A, Hallamm M, Smoot M, 2000 "Maxims or myths of beauty? A meta-analytic and theoretical review" Psychological Bulletin 126 390-423

Langlois J H, Roggman L A, 1990 "Attractive faces are only average" Psychological Science 1 115-121

Lewis K M, Robkin N, Gaska K, Njoki L C, 2011 "Investigating motivations for women's skin bleaching in Tanzania" Psychology of Women Quarterly 35 29-37

Lewis M B, 2010 "Why are mixed-race people perceived as more attractive?" Perception 39 136-138

Lie H C, Rhodes G, Simmons L W, 2008 "Genetic diversity revealed in human faces" Evolution 62 $2473-2486$

Lippman Z B, Zamir D, 2007 "Heterosis: revisiting the magic" Trends in Genetics 23 60-66

Little A C, Apicella C L, Marlowe F W, 2007 "Preferences for symmetry in human faces in two cultures: data from the UK and the Hadza, an isolated group of hunter-gatherers" Proceedings of the Royal Society of London B 274 3113-3117

Little A C, DeBruine L M, Jones B C, Waitt C, 2008 "Category contingent aftereffects for faces of different races, ages and species" Cognition 106 1537-1547

Little A C, Jones B C, DeBruine L M, 2011 "Facial attractiveness: Evolutionary based research" Philosophical Transactions of the Royal Society of London B 366 1638-1659

Marshall T C, Spalton J A, 2000 "Simultaneous inbreeding and outbreeding depression in reintroduced Arabian oryx" Animal Conservation $3241-248$

Mingroni M A, 2007 "Resolving the IQ paradox: Heterosis as a cause of the Flynn effect and other trends" Psychological Review 114 806-829

Penrose L S, 1955 "Evidence of heterosis in man" Proceedings of the Royal Society of London B 144 203-213

Penton-Voak I S, Jacobson A, Trivers R, 2004 "Populational differences in attractiveness judgements of male and female faces: Comparing British and Jamaican samples" Evolution and Human Behavior 25 355-370

Perrett D I, Lee K J, Penton-Voak I S, Rowland D R, Yoshikawa S, Burt D M, Henzi S P, Castles D L, Akamatsu S, 1998 "Effects of sexual dimorphism on facial attractiveness" Nature 394 884-887

Rhodes G, Lee K, Palermo R, Weiss M, Yoshikawa S, Clissa P, Williams T, Peters M, Winkler C, Jeffery L, 2005 "Attractiveness of own-race, other-race, and mixed-race faces" Perception 34 $319-340$

Roberts S C, Little A C, Gosling L M, Perrett D I, Carter V, Jones B C, Penton-Voak I, Petrie M, 2005 "MHC-heterozygosity and human facial attractiveness" Evolution and Human Behavior 26 213-226

Rowland D A, Perrett D I, 1995 "Manipulating facial appearance through shape and color" IEEE Computer Graphics and Applications 15 70-76

Setchell J M, Charpentier M J E, Abbott K M, Wickings E J, Knapp L A, 2010 "Opposites attract: MHC-associated mate choice in a polygynous primate" Journal of Evolutionary Biology $\mathbf{2 3}$ $136-148$

Suter S M, Keiser M, Feignoux R, Meyer D R, 2007 "Reed bunting females increase fitness through extra-pair mating with genetically dissimilar males" Proceedings of the Royal Society of London B $2742865-2871$

Tarvin K A, Webster M S, Tuttle E M, Pruett-Jones S, 2005 "Genetic similarity of social mates predicts the level of extrapair paternity in splendid fairy-wrens" Animal Behaviour 70 945-955 
Thornhill R, Gangestad S W, 1993 "Human facial beauty: averageness, symmetry, and parasite resistance" Human Nature 4 237-269

Tiddeman B P, Burt D M, Perrett D I, 2001 "Prototyping and transforming facial texture for perception research" IEEE Computer Graphics and Applications 21 42-50

Westermarck E, 1894 The History of Human Marriage (London: Macmillan)

Wolanski N, Jarosz E, Pyzuk M, 1970 "Heterosis in man — growth in offspring and distance between parents birthplaces" Social Biology 17 1-16

Wolf A P, 1993 “Westermarck Redivivus” Annual Review of Anthropology 22 157-175

\section{Appendix}

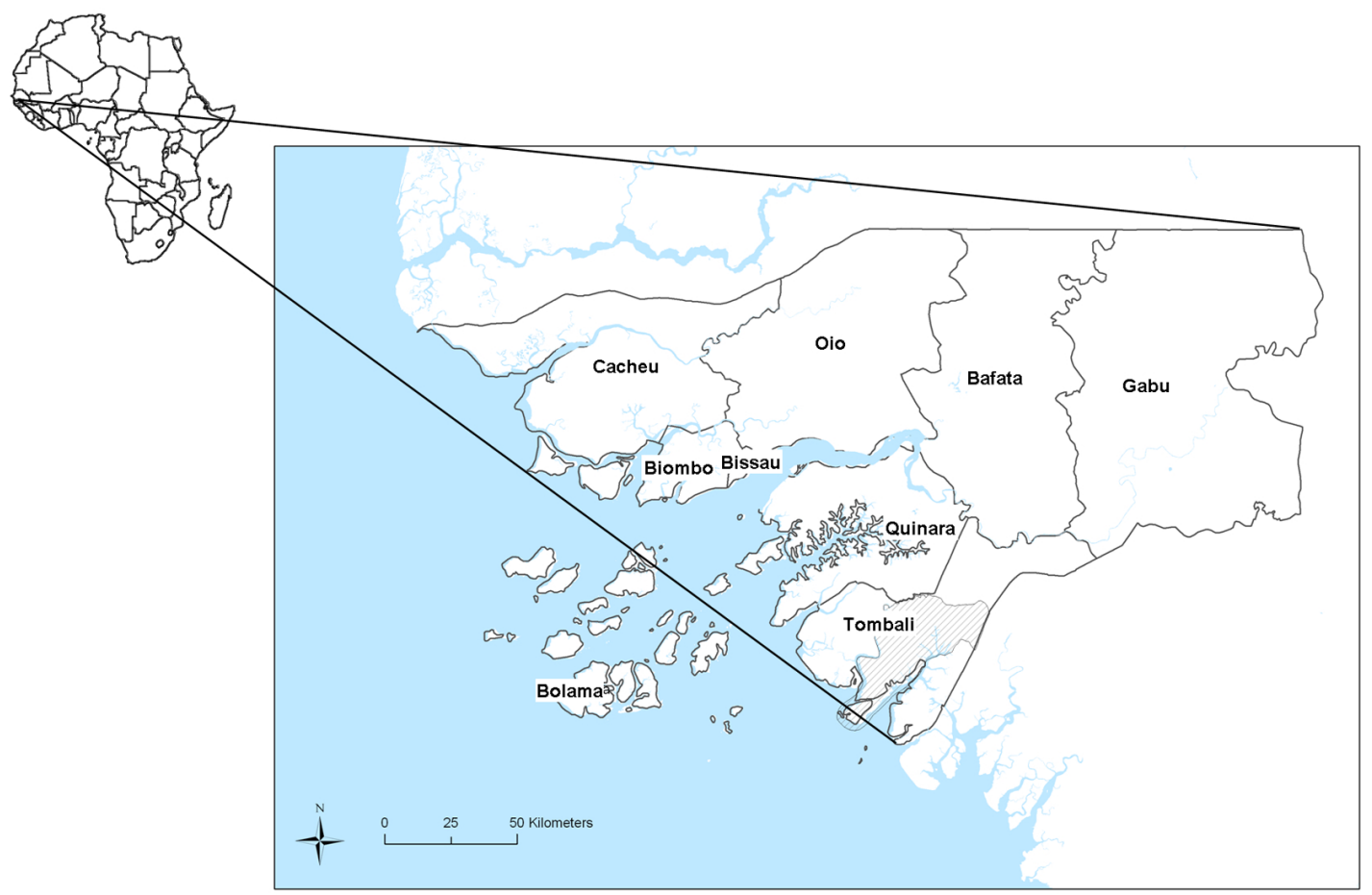

Figure A1. [In colour online.] Location of Guinea-Bissau in west Africa and Cantanhez National Park (highlighted with diagonal lines) in Tombali, Guinea-Bissau. Map created as part of project PPCDT/ ANT/57434/2004, financially supported by FCT, Portugal. 